\title{
LA INVESTIGACIÓN PSICOLÓGICA EN CAJAMARCA: UNA REVISIÓN BIBLIOGRÁFICA DE LOS ÚLTIMOS CINCO AÑOS
}

\author{
PSYCHOLOGICAL RESEARCH IN CAJAMARCA:
}

\author{
A BIBLIOGRAPHIC REVIEW OF THE LAST FIVE YEARS
}

\author{
Andy Rick Sánchez-Villena y Juan Pablo Sarmiento-Longo \\ Universidad Privada del Norte, Cajamarca, Perú
}

\begin{abstract}
Resumen
El presente trabajo tiene como principal objetivo analizar los trabajos de investigación psicológica realizados en Cajamarca desde el año 2014 hasta la actualidad. Para ello, se consideró un total de 135 investigaciones, los cuales se conforman por 113 tesis de grado y licenciatura y 22 artículos de revistas locales. Los resultados mostraron que la mayoría de investigaciones siguieron un diseño correlacional simple, utilizaron muestreo no probabilístico, reportaron la confiabilidad con el coeficiente alfa y no se reporta validez. Además, en los investigadores predomina el uso del SPSS.
\end{abstract}

Palabras clave: Investigación psicológica, revisión bibliográfica, Cajamarca.

\begin{abstract}
The main objective of this paper is to analyze the psychological research work carried out in Cajamarca from 2014 to the present. To achieve this aim, a total of 135 investigations were considered, which are made up of 113 graduate and undergraduate theses and 22 articles from two local journals. The results showed that the majority of investigations followed a simple correlation design, used non-probabilistic sampling, reported reliability with alpha coefficient and no validity was reported. Furthermore, the use of SPSS predominates among researchers.
\end{abstract}

Key words: Psychological research, bibliographic review, Cajamarca. 


\section{Introducción}

La investigación científica, es entendida como «el proceso de naturaleza compleja a través del cual se producen los conocimientos científicos» (Piscoya, 1995, p. 13). Gracias a estos conocimientos, el hombre ha podido modificar su entorno para responder a sus necesidades a fin de mejorar su calidad de vida (Bunge, 1959). En consecuencia, la investigación científica es importante porque a través de la aplicación del conocimiento generado se puede dar solución a los diversos problemas sociales, crear tecnología, innovar y mejorar la situación económica, técnica, política y cultural, especialmente en países en vías de desarrollo (Bunge, 1989) como el Perú. Por lo tanto, la investigación psicológica hace referencia a la actividad por la cual se estudian la conducta humana y los procesos cognitivos (Arana, Mellán y Pérez, 2006) haciendo uso del método científico para describirlos y/o explicarlos (Alarcón, 2013; Bunge, 1980); lo cual la convierte en una actividad más complicada debido a la naturaleza compleja de las personas que dan lugar a fenómenos como la violencia, la corrupción, la delincuencia, la agresión, las relaciones sociales, los vínculos familiares, entre otros.

La investigación en psicología, al igual que en otras ciencias, se puede clasificar en dos grandes tipos: básica y aplicada (Bunge, 1989; Piscoya, 1995; Sánchez y Carlessi, 2015). La primera refiere a aquella que busca aportar al conocimiento o a un cuerpo teórico, mientras que la segunda hace referencia a aquella que pretende dar solución a un problema determinado haciendo uso del conocimiento científico. En tal sentido, Alarcón (2013) refiere que «la ciencia no persigue únicamente fines teóricos, que quizá podrían alejarla de la realidad social y de los problemas humanos. Está también interesada en la modificación y dominio de la naturaleza para usarla en beneficio del hombre» (p. 36).

Mediante la investigación, los psicólogos también han jugado un papel importante y activo en el desarrollo de políticas enfocadas en los aspectos de salud y educación (Spink, 2009), un buen ejemplo de ello son los enfoques pedagógicos basados en teorías psicológicas como las de Jean Piaget, Albert Bandura, David Ausubel y Lev Vigotsky que son usadas en el ejercicio docente, especialmente a nivel de Educación Básica Regular (Ministerio de Educación [MINEDU], 2016)

Pero, además, la investigación psicológica ha contribuido al desarrollo de técnicas y tecnologías (Sánchez, 2016), prueba de ello son las diferentes técnicas psicoterapéuticas basadas en la evidencia (Labrador y Crespo, 2012; Llobell, Frías y Monterde i Bort, 2004), y los tests psicológicos, llámense cuestionarios, inventarios o escalas, siempre y cuando evidencien sus propiedades psicométricas mediante las herramientas estadísticas adecuadas. Al respecto, Alarcón (2002) señala que, dentro de las actividades investigativas en psicología, la psicometría es una de las más antiguas a nivel latinoamericano.

La psicología como profesión en el Perú data de 1955 cuando se creó la sección de Psicología en la Universidad Nacional 
Mayor de San Marcos cuya formación se vio influenciada por el psicoanálisis, lo cual generó que los psicólogos se enfocaran más en un trabajo clínico y psicoterapéutico y no a la investigación, lo cual sucede hasta hoy. Sin embargo, años más tarde, la investigación psicológica mejoró debido a la inserción de asignaturas como metodología, epistemología y estadística aplicada (Alarcón, 2012). No obstante, la investigación psicológica data de años anteriores, pues en 1935 se crea, a solicitud de Humberto Borja García en 1927, el primer laboratorio de psicología experimental en la misma universidad, el cual estuvo a cargo del Dr. Walter Blumenfeld (Alarcón, 2012, 2017). Aunque, Orbegoso (2015) puntualiza que éste no fue el primero, ya que, en 1916 la Escuela Normal de Varones contaba con un Gabinete de Psicología y posteriormente, en 1919, el Asilo de Alienados de Lima, hoy Hospital Víctor Larco Herrera, instaló otro laboratorio de psicología experimental.

A nivel universitario, como refiere Alarcón (2017), para la obtención del grado académico y la licenciatura, las universidades solían exigir una investigación al estudiante para que se le otorgue el grado de bachiller, pero «mediante Decreto Ley 739 del 8-IX-91, fue modificado dicho artículo disponiéndose que para obtener el grado de Bachiller basta la simple terminación de los estudios respectivos» (p. 17). Sin embargo, la situación ha cambiado, pues la Nueva Ley Universitaria $\mathrm{N}^{\circ} 30220$ (2014), señala en el artículo 45 que el grado de Bachiller, se obtiene con la aprobación de las asignaturas de pregrado además de un trabajo de investigación. De igual manera, el Título profesional requiere la aprobación de una Tesis o un trabajo de suficiencia profesional, de esta manera se anulan los cursos de titulación. Así, la Ley Universitaria $\mathrm{N}^{\circ} 30220$ (2014) hace hincapié en que la universidad debe ser una institución centrada en la investigación, por lo que los docentes deben ser investigadores. Sin embargo, la realidad difiere de lo estipulado en la ley, pues son pocos los docentes, específicamente en psicología, que se dedican a esta actividad y que son reconocidos por CONCYTEC. Al respecto Sánchez (2016) refiere que hasta agosto del 2016 hubo 48511 profesionales registrados en el Directorio de Recursos Humanos afines al CTI (DINA) de los cuales 655 (1,35\%) eran psicólogos. El $54,50 \%$ (357) de ellos provenían de Lima, de éstos solo el 4,12\% (27) estaban registrados en el Registro de Investigadores en Ciencia y Tecnología del Sistema Nacional de Ciencia, Tecnología e Innovación Tecnológica (REGINA), de los cuales 21 eran de Lima. Cabe señalar que de los 6 psicólogos restantes calificados por CONCYTEC, ninguno era de Cajamarca y la situación sigue siendo igual.

Lo anterior evidencia la incipiente cultura de investigación en el área psicológica, en especial en Cajamarca, ya que las universidades no cuentan con docentes calificados en la enseñanza de la investigación psicológica. Esto provoca que, a pesar del grado académico, la investigación no les genere mayor interés debido a que no consideran la actividad científica como parte de su labor docente o profesional (Bermúdez, 2013). A su vez influye en ideas erradas o 
distorsionadas que tienen los estudiantes de psicología respecto a la investigación (Sánchez-Vásquez, 2014) y en retrasos al momento de llevar a cabo su tesis de grado o licenciatura. Lo anterior explicaría el por qué en Cajamarca, no existe producción científica permanente en psicología aparte de las tesis de licenciatura exigidas por Ley, pues, además, solo se cuenta con una revista especializada en psicología llamada Tendencias en Psicología, editada por la Universidad Privada Antonio Guillermo Urrelo, la primera universidad privada de Cajamarca y la primera en ofrecer la carrera de Psicología desde hace 20 años (Sarmiento, 2008), la cual cuenta con 1 volumen, 2 números, 13 artículos y data del 2016; sin embargo no está indexada y no se han realizado más publicaciones desde ese año. Esta misma universidad cuenta con otra revista multidisciplinaria indexada en Latindex llamada Perspectiva, que incluye publicaciones de autores locales desde el 2014 referidas a variables psicológicas, pero no todos los autores son psicólogos (los resultados se describirán y discutirán con más detalle en el apartado correspondiente).

Ante tal situación, el presente trabajo tiene como principal objetivo analizar los trabajos de investigación psicológica en Cajamarca y tener una visión general de lo que se ha realizado en esta área a partir de la vigencia de la Nueva Ley Universitaria $\mathrm{N}^{\circ} 30220$ (2014) que exige una investigación para obtener cualquier grado académico y otra para el título profesional, lo cual permite analizar la tendencia de la investigación psicológica en Cajamarca para responder y discutir preguntas como: ¿Qué variables son las más estudiadas?, ¿qué enfoque es el más frecuente?, ¿qué métodos estadísticos son los más usados?, ¿qué diseños metodológicos se han usado?, ¿qué tipo de muestra se usa más?, ¿qué instrumentos han sido los más usados?, ¿se han reportado adecuadamente sus propiedades psicométricas?, ¿qué programa estadístico se usa con más frecuencia?, ¿qué carencias o deficiencias presentan las investigaciones en Cajamarca?, entre otras de importancia metodológica en la investigación psicológica. Además, se considera que los resultados podrán aportar a la literatura científica local, a mejorar y a divulgar lo que se está haciendo al momento de investigar en psicología en esta parte del país.

\section{Método}

\section{Selección de estudios}

Para realizar la presente investigación se consideraron todos los trabajos de grado y titulación en psicología que se encuentran en los repositorios digitales de la Universidad Privada Antonio Guillemo (UPAGU) y de la Universidad Privada del Norte - Sede Cajamarca (UPN-C) a partir del 2014 hasta enero del 2019, puesto que son las únicas universidades en Cajamarca que ofrecen la carrera profesional de Psicología actualmente.

Por otro lado, se consideraron todos los artículos publicados en la Revista Tendencias en Psicología (por tratarse de una revista especializada en aspectos psicológicos), los artículos con variables psicológicas de la revista Perspectiva, la 
cual es una revista multidisciplinaria de UPAGU donde se aceptan investigaciones psicológicas, y un artículo publicado en la revista Eureka de Paraguay por tratarse de un autor proveniente de Cajamarca (Sánchez-Villena, 2018).

Como criterios de inclusión de las tesis para optar un grado académico o título profesional se tuvieron en cuenta: 1 ) investigaciones empíricas, teóricas o instrumentales; 2 ) que sean de pregrado, maestrías de psicología clínica o familia, y que 3) hayan sido presentadas a partir del 2014.

Respecto a los criterios de inclusión para las publicaciones en las revistas se consideraron: 1) que los artículos sean investigaciones empíricas, teóricas o instrumentales; 2) que los artículos tengan por lo menos una variable psicológica en el ámbito clínico, organizacional, educativo o social;3) que hayan sido publicadas a partir del año 2014; y 4) que sus autores sean locales.

La búsqueda y recopilación se dividió en cuatro etapas: primero se identificaron las tesis de psicología de pregrado tanto en el repositorio digital de UPAGU como de UPN-C. En segundo lugar, se identificaron las tesis de postgrado (maestría en psicología clínica y maestría en familia) de UPAGU. En tercer lugar se recopilaron los artículos publicados en la revista Tendencias en Psicología junto a la publicación en la revista Eureka; y finalmente se identificaron los artículos con variables psicológicas publicadas en la revista Perspectiva, cuyas publicaciones fueron sometidas a los criterios de inclusión.

\section{Codificación de los datos}

Para codificar los datos se hizo uso del programa Microsoft Excel 2016 donde se crearon dos hojas de cálculo, una para crear la base de datos de las tesis de pregrado y postgrado y una segunda para crear una matriz con los artículos en las revistas ya mencionadas.

En el caso de las tesis se obtuvo información del título, autor, año de publicación, variables, diseño de investigación, enfoque, cantidad de muestra, tipo de muestra reportada, instrumentos utilizados, validez, confiabilidad, pruebas estadísticas y software utilizado. Mientras que para las revistas se agregó, además de la información anterior, la indexación.

\section{Resultados}

Se identificó un total de 134 investigaciones de grado y licenciatura: 73 tesis fueron para optarporel títuloprofesional de licenciado(a) en psicología (cincuenta y uno en UPAGUy veintiuno en UPN-C) yuna monografía para el grado académico de Bachiller en UPAGU, la cual fue descartada por incumplirel primer criterio de inclusión. En las investigaciones de postgrado, se identificaron 61 tesis (treinta y ocho de maestría en psicología clínica, tres de maestría en familia, once de maestría en administración, seis en gestión de la educación, y tres en doctorado), de las cualesveintefueron descartadas debidoaque no eran tesis de psicología clínica ni familia. Dando así, un total de 113 tesis.

Respecto a los artículos se identificaron 34 (treceen la revista Tendencias en Psicología, 
uno en Eureka y veinte en Perspectivas), de los cuales se descartaron doce. Nueve por ser colaboración internacional, uno porque el autor no es local, y dos por ser ensayos. En consecuencia, se obtuvieron 22 artículos, lo cual, sumado a las 113 tesis mencionadas, hacen un total de 135 investigaciones psicológicas en Cajamarca.

\section{Tesis de grado y licenciatura}

Año de publicación de tesis

de grado y licenciatura

Respecto al año en que fueron realizadas las tesis de grado y licenciatura, treinta y ocho $(33,6 \%)$ se hicieron en el 2018, veintiocho $(24,8 \%)$ en el 2017 , veinticinco $(22,1 \%)$ en el 2016 y veintidós $(19,5 \%)$ en el año 2015.

Variables estudiadas en tesis

de grado y licenciatura

Se encontraron 94 diferentes variables, siendo la más estudiada la inteligencia emocional con once tesis $(5,6 \%)$, seguida de la autoestima con nueve tesis $(4,6 \%)$, la agresividad con ocho tesis $(4,1 \%)$, el clima social familiar con siete tesis (3,6\%), depresión con siete tesis $(3,6 \%)$, síndrome de burnout con siete tesis $(3,6 \%)$, bienestar psicológico con seis tesis $(3,1 \%)$, ideación suicida con seis tesis $(3,1 \%)$ y rendimiento académico con seis tesis (3,1\%).

\section{Diseño y enfoque de investigación}

en tesis de grado y licenciatura

Respecto al diseño de investigación teniendo en cuenta la clasificación de Ato, Lópezy Benavente (2013), se encontraron ocho diferentes diseños. De las 113 tesis de grado y licenciatura, setenta y ocho (69\%) correspondían al diseño correlacional simple, dieciséis $(14,2 \%)$ eran de grupos naturales, seis $(5,3 \%)$ seguían un diseño selectivo no probabilístico transversal, cuatro $(3,5 \%)$ tenían un diseño con grupos no equivalentes, cuatro $(3,5 \%)$ tenían un diseño selectivo probabilístico transversal, tres $(2,7 \%)$ eran estudios instrumentales, uno $(0,9 \%)$ seguia un diseño pretest postest y uno $(0,9 \%)$ era un estudio correspondiente a un diseño observacional. De las anteriores, ciento doce $(99,1 \%)$ se enmarcaban dentro del paradigma cuantitativo, mientras que el estudio restante $(0,9 \%)$ se encontraba dentro del paradigma cualitativo.

Muestra en tesis de grado y licenciatura

Del total de tesis de grado y licenciatura, cincuenta y uno (45\%) utilizaron un muestreo no probabilístico en sus diversos tipos, treinta y siete (33\%) no reportaron el tipo de muestreo, dieciocho (16\%) utilizaron el muestreo probabilístico en sus diferentes tipos, y siete (6\%) consideraron trabajar con la totalidad de su población. No obstante, solo treinta y seis $(31,9 \%)$ estudios reportaron sustento bibliográfico al respecto. La cantidad de participantes mínima fue de 10 y un máximo de 987.

Instrumentos en tesis de grado y licenciatura

En cuanto a los instrumentos utilizados para la recolección de datos, se encontraron 127 diferentes escalas, cuestionarios, inventarios o test. Los más utilizados 
fueron el Inventario de Inteligencia Emocional de BarOn, utilizado en ocho estudios (4,2\%), seguido del Inventario de Burnout de Maslach en seis estudios (3,1\%), el Cuestionario AQ de Agresividad de Buss y Perry, utilizado en cinco estudios $(2,6 \%)$ al igual que la Escala de Autoestima de Coopersmith (2.6\%), y la Escala FES de Clima Social Familiar (2,6\%). La Escala de Bienestar Psicológico de Ryfffue utilizada en cuatro estudios $(2,1 \%)$ y el AUDIT en otras cuatro tesis $(2,1 \%)$.

Del total de instrumentos empleados, cincuenta y dos (46\%) no reportaron la validez del instrumento. Veintidós $(19,5 \%)$ mencionaron la validez de constructo con Análisis Factorial Exploratorio (AFE) (un estudio instrumental). Diecisiete $(15 \%)$ reportaron solo validez de contenido. Doce $(10,6 \%)$ reportaron validez convergente. Cinco $(4,4 \%)$ reportaron Análisis Factorial Confirmatorio (AFC). Dos $(1,8 \%)$ mostraron AFE junto a AFC (estudios instrumentales). Dos solo mostraron la correlación ítem-test (2\%). Y el estudio restante reportóvalidez predictiva $(0,9 \%)$.

Cabe señalar que de los estudios empíricos que mencionaron al AFE, tres evidenciaron únicamente los valores obtenidos por la prueba Kaiser-Meyer-Olkim y la prueba de esfericidad de Barlett. Dos mostraron adecuadamente el procedimiento del AFE. Y de los estudios restantes se desconocen si los AFE fueron hechos en un contexto local, pues se encontró, en algunas tesis, que la redacción de la sección validez y confiabilidad eran similares a las de otros estudios. Otros solo mencionaron el AFE, pero no describieron el procedimiento (el método de extracción, rotación, varianza explicada y factores extraídos) o no hay evidencias de haberlo ejecutado. Además, de los cinco estudios empíricos que reportaron AFC, cuatro fueron realizados en otros contextos y uno es dudoso, pues se halló plagio en la sección de validez y confiabilidad.

Respecto a la confiabilidad de los instrumentos, ochenta y cuatro $(74,3 \%)$ utilizaron el coeficiente alfa, dieciocho $(15,9 \%)$ no reportaron la confiabilidad, cinco $(4,4 \%)$ reportaron el método de dos mitades. Dos (1,8\%) reportaron el coeficiente alfa junto al KR2o. Uno (o,9\%) reportó el coeficiente alfa junto al método de dos mitades, otro reportó coeficiente alfa y test- retest $(0,9 \%)$. Uno más reportó solo el KR2o $(0,9 \%)$ y el restante reportó solo test-retest $(0,9 \%)$.

\section{Pruebas estadísticas en tesis}

de grado y licenciatura

Como se ha referido anteriormente, de las 113 tesis de grado y licenciatura, tres $(2,7 \%)$ fueron instrumentales, las cuales hicieron uso de AFE y el coeficiente de correlación de Pearson, y solo dos usaron AFC.

Teniendo en cuenta solamente los estudios empíricos, se encontraron 16 estadísticos diferentes usados 160 veces. Los más usados fueron la prueba de normalidad Kolmogorov-Smirnov, reportado treinta y un veces (19,4\%). El coeficiente de correlación de Spearman utilizado treinta veces $(18,8 \%)$. El coeficiente de correlación de Pearson fue utilizado veintidós veces $(13,8 \%)$. El Chi-cuadrado de Pearson fue utilizado veintiún veces $(13,8 \%)$. La 
$\mathrm{t}$ de Student fue reportada trece veces $(8,1 \%)$. La U de Mann Whitney fue utilizada once veces $(6,9 \%)$. Cabe resaltar que las tablas de frecuencias junto a los gráficos de barras o gráficos circulares fueron utilizados otras once veces $(6,9 \%)$, correspondientes a todas las investigaciones con diseños selectivos y a un estudio con diseño de grupos naturales.

Software en trabajos de grado y licenciatura

De los 113 trabajos de grado y licenciatura, noventa y nueve $(87,6 \%)$ usaron el Statistical Package for the Social Sciences (SPSS) en sus diferentes versiones como software estadístico para analizar sus datos. Once $(9,7 \%)$ no reportaron el programa de análisis de datos. Dos $(1,8 \%)$ utilizaron SPSS y AMOS de manera conjunta. Mientras que el estudio restante, que corresponde a la investigación observacional, no especificó si hizo uso, o no, de algún programa.

\section{Artículos}

\section{Características}

De los 22 artículos, quince $(68,2 \%)$ fueron publicados en el 2016, cuatro $(18,2 \%)$ se publicaron en el 2017 , y veintidós fueron publicados en el 2018. En cuanto a la revista, once (50\%) se publicaron en Tendencias en Psicología, la cual no está indexada. Diez (45,5\%) se publicaron en Perspectiva, la cual se halla indexada solo en Latindex, y una en Eureka (4,5\%), la cual está indexada en Web of Science. Asimismo, la revista Tendencias en Psicología pertenece al área de psicología al igual que Eureka, mientras que la revista Perspectiva es multidisciplinaria y se publican artículos con temas provenientes de diferentes áreas como las Ciencias Empresariales y Administrativas, Derecho, Ciencias de la salud, Ingeniería y Psicología.

\section{Variables estudiadas}

Se encontraron 29 diferentes variables estudiadas y 2 temas de interés correspondientes a dos investigaciones teóricas. Lo más estudiado fue el compromiso organizacional considerado cuatro veces $(13,8 \%)$, el clima organizacional, considerado dos veces $(6,9 \%)$, la motivación laboral, estudiado dos veces $(6,9 \%)$, y los estilos de crianza, estudiados dos veces $(6,9 \%)$.

\section{Diseño y enfoque de investigación}

De los 22 artículos, considerando la clasificación de Ato, Lópezy Benavente (2013), once $(50 \%)$ seguían un diseño correlacional simple. Dos (9,1\%) seguían un diseño selectivo transversal y dos (9,1\%) eran investigaciones teóricas. El resto seguía un diseño con grupos no equivalentes $(4,5 \%)$, diseño pretest-postest $(4,5 \%)$, diseño retrospectivo ex post facto $(4,5 \%)$, grupos naturales $(4,5 \%)$, observacional $(4,5 \%)$, predictivo transversal $(4,5 \%)$ y un diseño selectivo no probabilístico $(4,5 \%)$. Además, solo seis $(27,3 \%)$ artículos mostraron sustento bibliográfico.

\section{Muestra}

Del total de artículos, diez $(45,5 \%)$ no especificaron ni reportaron el tipo de 
muestreo utilizado. Siete $(31,8 \%)$ reportaron muestreo no probabilístico en sus diferentes tipos y tres $(13,6 \%)$ reportaron un muestreo probabilístico. Los dos restantes (9,1\%) no reportaron muestra, pues eran estudios teóricos. Además, diecinueve $(86,4 \%)$ artículos no reportaron sustento bibliográfico. La cantidad mínima de participantes en la muestra fue de 8 y un máximo de 352 .

\section{Instrumentos}

Se encontraron 35 diferentes instrumentos. La Escala de Estilos de Socialización Parental (ESPA-29) fue utilizada dos veces. El resto de instrumentos fueron utilizados una sola vez. Incluso, hay un artículo de la revista Perspectiva con dos instrumentos $(5,7 \%)$ que no están especificados, pues refiere que usó cuestionarios y una encuesta.

Excluyendo los dos estudios teóricos, dieciséis $(80 \%)$ no reportaron ningún tipo de validez, y dos (10\%) reportaron solo validez de contenido y uno (5\%) reportó AFC. Respecto a la confiabilidad, diez (50\%) no la reportaron ni la especificaron, seis (30\%) reportaron el coeficiente alfa, dos $(10 \%)$ reportaron la confiabilidad mediante el método de dos mitades, uno (5\%) reportó el coeficiente alfa y el coeficiente omega. Y el artículo restante no evidenció validez ni confiabilidad, pues fue observacional y utilizó entrevistas.

\section{Pruebas estadísticas}

Respecto a las pruebas estadísticas, se encontraron once estadísticos diferentes utilizados veintisiete veces en los estudios empíricos excluyendo a los dos teóricos y al observacional.

El coeficiente de correlación de Pearson fue utilizado cinco veces $(18,5 \%)$, la estadística descriptiva junto a las frecuencias y/o gráficos fueron reportadas cinco veces $(18,5 \%)$ y la prueba de normalidad fue utilizada cuatro veces $(14,8 \%)$. La U de Mann Whitney fue usada tres veces $(11,1 \%)$ y la $t$ de Student fue utilizada dos veces $(7,4 \%)$. El Chi-cuadrado (3,7\%), ANOVA $(3,7 \%)$, Kruskall Walis $(3,7 \%)$, regresión lineal múltiple (3,7\%), Shapiro Wilk $(3,7 \%)$ y el coeficiente de correlación de Spearman (3,7\%) fueron utilizadas una vez.

\section{Software}

Excluyendo a los artículos teóricos y al observacional, que no hicieron uso de software para el análisis de datos, nueve $(47,4 \%)$ usaron el SPSS. Otros nueve $(47,4 \%)$ no reportaron ningún software estadístico y uno $(5,2 \%)$ señaló haber utilizado Microsoft Excel.

\section{Discusión}

La presente investigación constituye una revisión bibliográfica de la investigación en Cajamarca desde el año 2014. Dentro de ésta, se han considerado trabajos de grado y licenciatura y artículos publicados en dos revistas científicas locales. El objetivo del estudio es analizar las publicaciones y tesis de psicología para tener un panorama global acerca de la tendencia en investigación en esta parte del país, lo 
que permite, también, identificar carencias o limitaciones al momento de hacer investigación psicológica a fin de plantear mejoras y superar obstáculos, principalmente, metodológicos o estadísticos con sustento en la evidencia.

La investigación psicológica cajamarquina es incipiente, considerando que solo la Universidad Privada Antonio Guillermo Urrelo, era la que ofertaba la carrera de psicología desde el año 1999, siguiendo sus funciones hasta la actualidad. En caso de la Universidad Privada del Norte, con sede principal en Trujillo, creó una sede en la ciudad de Cajamarca en el año 2005, pero la carrera de Psicología recién se instauró en 2010.

Es importante detallar el contexto nacional respecto a la investigación. La investigación psicológica en el Perú no ha llegado a consolidarse al nivel de desarrollo de otras ciencias, pues se replica y continua con investigaciones del siglo XX (Sánchez, 2016). Además, esto se une a la tendencia dependientey como consecuencia, falta de originalidad en las propias investigaciones (Alarcón, 2002). Si bien es cierto, esta idea se planteaba respecto a la investigación en el Perú y Latinoamérica, es coherente con lo que ocurre en Cajamarca, ya que las investigaciones, especialmente las instrumentales, se centran en estudios asociados a la adaptación de instrumentos elaborados en otras realidades, antes que en la creación de escalas de medición que respondan y se adecúen al contexto nacional o local. Además, se sabe que, en la realidad peruana, la investigación tuvo auge entre 1970 y 1990 por la presencia de universidades que ofertaban la carrera de Psicología y que tenían, como mecanismo para obtener el bachillerato y la licenciatura, la presentación de una tesis. Sin embargo, al eliminarse este último requisito para obtener el bachillerato, disminuyó la cantidad de tesis, haciendo que la Psicología pierda su carácter científico primario, anteponiéndose la actividad profesional (Sánchez, 2016). Ahora bien, los pocos años de desarrollo de la Psicología en Cajamarca como formación académica, se refleja en la cantidad de investigaciones en comparación a otras ciudades. Además, los mecanismos para obtener títulos profesionales han establecido nuevos parámetros, ya que anteriormente se podía obtener la licenciatura mediante cursos de titulación, lo que iba en desmedro de la cantidad de tesis de pregrado. Sin embargo, las nuevas políticas de SUNEDU, han influenciado en la mayor cantidad de tesis de pregrado, evidenciándose un incremento en los últimos cinco años.

Los resultados del presente trabajo reflejan, de manera general, el desinterés por divulgar los resultados de las investigaciones de grado o licenciatura y someterlos a revisión por pares en revistas indexadas en bases de datos bibliográficas importantes como Scopus o Web of Science, incluso a nivel de posgrado, donde la investigación es el eje fundamental y se forman investigadores. Ante ello, Bermudez (2013) sostiene que «si los resultados de las investigaciones no se publican o no se divulgan para conocimiento, análisis, validación o refutación en el ámbito de la comunidad científica, es como si no se hubieran realizado» (p. 15). 
Este fenómeno podría deberse a la falta de experiencia en la elaboración de una tesis o de cualquier investigación, pues esto demanda compromiso, tiempo e implica hacer uso de recursos cognitivos complejos para construir, por ejemplo, el marco teórico, redactar según criterios científicos, y tener conocimiento en estadística cuando se trabaja dentro del enfoque cuantitativo (Ochoa, 2011); lo cual provoca percepciones negativas e ideas erradas en los estudiantes respecto a la actividad investigativa (Sánchez-Vásquez, 2014).

Considerando los temas o las variables de estudio, se han considerado los estudios con variables individuales, es decir, aquellas que permiten determinar características de los sujetos sin necesidad del grupo. Dentro de este tipo de variables, se encuentran aquellas de corte clínico; además, que incluyen las habilidades sociales, autoestima y otras variables que evalúan el desarrollo individual. Una tendencia importante es el estudio de variables de corte familiar, algo que fomenta el área que incluye la profundización del estudio del grupo más importante para la socialización de todo individuo. Este hecho puede deberse a que, dentro de las universidades locales, se imparten cursos que profundizan en el enfoque sistémico antes que otras corrientes psicológicas, que si bien no necesariamente se centra en la terapia familiar o de pareja, se le ha dado dicho carácter. Al respecto, se hace necesario recordar lo que plantea Sánchez (2016) quien considera que diversos problemas teóricos de la Psicología no se exploran, como la conciencia, la comprensión, la voluntad, etc. Además de problemas de la psicología negativa como el resentimiento, la agresividad, la violencia psicosocial, comportamiento social negativo, envidias, motivación de poder, maltrato familiar, violación sexual, entre otros.

Respecto al año de las investigaciones, los resultados muestran un incremento en la cantidad de las tesis de grado y licenciatura, pues en el 2015 se publicaron veintidós tesis, veinticinco en $\mathbf{2 0 1 6}$, veintiocho en 2017 y treinta y ocho en el 2018, lo que indicaría un mayor número de egresados de Psicología y maestros en Psicología Clínica y Familia. Sin embargo, esto no implicaría un aumento en el interés por la investigación, ya que la Nueva Ley Universitaria $\mathrm{N}^{\circ} 30220$ (2014) indica que los egresados deben realizar una tesis para optar por la licenciatura y/o por algún grado académico. De manera opuesta, se observó una tendencia decreciente en la publicación de artículos, ya que el 2016 fue el año con mayores publicaciones en revistas $(68,2 \%)$, mientras que en 2017 se publicaron cuatro $(18,2 \%)$ y en 2018 solo se hallaron tres $(13,6 \%)$ publicaciones. Esto podría deberse a que en 2017 dejó de publicarse la única revista especializada en psicología de Cajamarca, en la cual los estudiantes tenían oportunidad de publicar artículos teóricos, empíricos o ensayos con temas de interés psicológico. No obstante, lo anterior también podría considerarse un indicador de la falta de interés por la difusión de los resultados, pues bien se podría publicar en otras revistas nacionales o internacionales, pero no se observó más que una sola publicación en una revista de Paraguay. 
El punto positivo es la diversidad de instrumentos utilizados, sin embargo, debe haber cuidado, pues el investigador no se puede regir al uso de un instrumento en su realidad como único criterio para su aplicación, pues es necesario identificar que las propiedades psicométricas sean las adecuadas y se adapten a las características propias de la población objetivo, de lo contrario, los resultados podrían verse sesgados, principalmente en estudios comparativos (de grupos naturales), pues muchos instrumentos no reportan la invarianza factorial. Dentro de este aspecto, es importante señalar que el $46 \%$ de los instrumentos aplicados en las tesis de grado o licenciatura no reportaron la validez del instrumento y el 19,5\% lo hizo haciendo uso del Análisis Factorial Exploratorio, de los cuales tres reportaron solo la prueba de Esfericidad de Barletty el $\mathrm{KMO}$, incluso otros fueron un copy-paste de la sección de validez del manual o de otras fuentes, donde no fueron halladas las propiedades psicométricas para el contexto local. Así, solo dos tesis reportaron adecuadamente el procedimiento del AFE. De los demás estudios se desconoce si fueron realizados en Cajamarca. En cuanto al AFC, cinco tesis lo reportaron, pero cuatro provenían de otras regiones y uno es dudoso, ya que se halló plagio en el apartado de validez y confiabilidad.

Por otro lado, respecto a la confiabilidad, ochenta y cuatro $(74,3 \%)$ tesis y seis (30\%) artículos reportaron el mal llamado Alfa de Cronbach (Cronbach y Shavelson, 2004), pues deberíamos llamarlo solo coeficiente Alfa. Lo anterior podría ser un indicador el abuso de este coeficiente, pues los supuestos para usarlo son: a) la tau-equivalencia, b) que la escala de los ítems sea continua y con distribución normal, c) que los errores de los ítems no covarien y d) que la escala sea unidimensional (McNeish, 2017). Sin embargo, muchos de los instrumentos no cumplen con estos criterios. Incluso se ha reportado un coeficiente Alfa para la escala total, cuando el instrumento mostraba multidimensionalidad, incumpliendo el cuarto supuesto. La explicación de este hecho reside, en primer lugar, en el desconocimiento de aspectos de psicometría en Cajamarca; y, en segundo lugar, al manejo exclusivo del SPSS para el análisis de datos, el cual tiene por defecto el coeficiente Alfa. Por esta razón, es necesario sugerir el uso de programas estadísticos más completos que brinden coeficientes más pertinentes como JASP (JASP Team, 2018), Jamovi (Jamovi Project, 2018) o R (R Core Team, 2018).

Los tres estudios instrumentales merecen una discusión particular, pues todos ellos llevaron a cabo el AFE mediante el SPSS. Sin embargo, se sabe que este hace uso de matrices de correlación de Pearson y no brinda la opción de trabajar con correlaciones policóricas o tetracóricas en el caso de items dicotómicos, al menos no en su configuracion predeterminada, lo cual no siempre es la opción correcta (Ferrandoy Lorenzo-Seva, 2014), más aun cuando se extraen factores mediante rotación varimax y el método de componentes principales, cuyo uso está actualmente desaconsejado (Lloret-Segura, Ferreres-Traver, Hernández-Baeza y Tomás-Marco, 2014). Para ello, se sugiere el uso de programas 
especializados como FACTOR (LorenzoSeva y Ferrando, 2006). Por otro lado, para los dos AFCs reportados se hizo uso de Máxima Varosimilitud (ML) como estimador mediante el software AMOS, considerando a las variables como continuas y normales, cuando bien pudieron presentar no normalidad y tratarlas como ordinales. Ante ello sería recomendable el reporte de la normalidad multivariada, la cual se puede calcular mediante el coeficiente de Mardia (Mardia, 1970) y el uso de estimadores adecuados como ULS o estimación Bayesiana en AMOS (Byrne, 2010), DWLS en LISREL o WLSMV en Mplus o a través del paquete Lavaan en R.

En psicología, los enfoques cuantitativo y cualitativo, tienen la similitud que siguen un método que derivará en una discusión analítica (García, 2015), sin embargo, existen diferencias que pueden marcar la tendencia de los investigadores para orientarse por uno de ellos. En principio, la psicometría permite dar un valor numérico a las variables psicológicas. $\mathrm{Al}$ respecto, García (2015) considera que nos permite tener un mayor conocimiento sobre una presunta existencia de algunos fenómenos, además de generar un lenguaje uniformado, necesario en toda investigación. Dentro de ellos, el enfoque cuantitativo es el más popular entre las investigaciones revisadas. Tal vez, debido al bajo nivel de complejidad que conlleva la ejecución de las investigaciones, por ejemplo, correlacionales, descriptivas o comparativas. Es importante considerar este punto, pues gran parte de los estudios revisados son tesis para alcanzar algún grado académico y lo que se requiere es rapidez para obtenerlos. Por lo tanto, la cantidad de estudios cuantitativos, respondería a intereses pragmáticos, políticos, mediáticos, comerciales o sociales (Sánchez, 2016). Lo cual es comprensible si se tiene en cuenta que, ante la competencia profesional, los egresados de las universidades busquen la forma de investigar que les tome menos tiempo. La otra razón, considerando la cantidad de estudios cualitativos, es que este enfoque es poco desarrollado, y por ende, al no haber investigaciones al respecto, no permite tener referencia para la elaboración de estudios de este enfoque en la realidad local.

En el caso de los diseños de investigación, los resultados mostraron que once artículos $(50 \%)$ y setenta y ocho (69\%) tesis de grado y licenciatura tenían un diseño correlacional simple, seguido del diseño degrupos naturales y selectivo transversal. Los estudios correlacionales simples, se caracterizan por no tener control de terceras variables y, en consecuencia, la validez externa e interna del estudio puede verse afectada (Ato, López y Benavente, 2013), y los diseños que buscan comparar grupos o describir algún fenómeno, generalmente, evalúan una sola variable. Esta tendencia la marca Sánchez (2016), al considerar que existen investigaciones extremadamente cuantitativas, descriptivas y correlacionales, algunas comparativas y muy pocos causales o explicativos. Esta elección por parte de los estudiantes, tanto en pregrado como en postgrado, bien podría reflejar la necesidad de elaborary ejecutar de manera más sencilla la investigación para obtener su grado académico o título profesional sin 
mostrar mayor interés en desenvolverse en el campo científico y en el desarrollo de una investigación que pueda solucionar un problema real o producir tecnología para ser usada en el campo de la psicología como las pruebas psicológicas o programas psicológicos (Sánchez, 2016), lo cual se ve reflejado en los escasos estudios instrumentales, pues solo se reportaron tres como trabajos de grado, o en las ocho investigaciones de diseño con grupos equivalentes y pretest-postest. Esto entra en contraste a la tendencia que había en Perú hace más de 50 años, ya que en la época de los 40 y 50 era frecuente la adaptación y validación de instrumentos (Alarcón, 2002), sin embargo, la disminución de investigación y el poco conocimiento sobre validación de los mismos, origina que la tendencia actual sea baja, en comparación con otro tipo de estudios, especialmente en el contexto local. Si bien es cierto, cualquier tipo de investigación aporta a la ciencia, los estudios de caso, son especialmente importantes para la Psicología. Los experimentales requieren mayor participación del investigador al manipular o administrar alguna variable en contraparte con los no experimentales, en los que el investigador tiene una actitud más bien pasiva respecto a la observación de las variables (García, 2015). Un buen grupo de investigaciones revisadas son de maestría, que en forma, deberían tener mayor rigurosidad o complejidad en comparación con los estudios de pregrado pero Sánchez (2016) considera que las tesis, en cantidad no son significativas y en calidad no son destacables ya que siguen líneas de trabajo y orientaciones del siglo XX.
Lo anterior también está relacionado con el uso de los diferentes estadísticos. Se sabe que la estadística nos permite organizar, sintetizar, reduciry generalizar observaciones. Además, en la actualidad existen diversos programas que nos permiten procesar los datos de una manera ordenada (García, 2015). La diversidad de estadística es llamativa, sin embargo, es importante recalcar que esto puede ser contraproducente en el sentido de la no existencia de criterios para hacer los análisis correctamente. Así, los coeficientes de correlación de Pearson, Spearman, Chi cuadrado, la t de Student y la U de Mann Whitney han sido los más utilizados para lograr los objetivos de las investigaciones en Cajamarca. Al respecto, cabe señalar que gran parte de las tesis y artículos analizados concluyen sus resultados haciendo uso únicamente del test de significancia estadística (NHST), y por lo tanto utilizan la palabra significativa(s) junto a correlación o diferencias dando a entender que sus resultados indican relevancia o es, como refiere Pita y Pértega (200o) una «garantía de calidad» (p. 191), cuando se sabeque en realidad, señalan probabilidad y dependen del tamaño de la muestra (Sarria y Silva, 2004; Ventura-León, 2017). Por ello, NHST ha sido criticado ampliamente, así Rozeboom (1960) refiere que esta es una práctica transmitida a través de la enseñanza de los cursos universitarios que se ha vuelto una costumbre calificada como un «ritual tribal para el procesamiento de datos» (p. 417), dando a entender su obsolescencia. Por ello, como un método alternativo a la prueba de hipótesis, la American Psychological Association [APA] (2010) exige el reporte 
de tamaños de efecto (TE). No obstante, en la realidad local se ha visto solo una $\mathrm{d}$ de Cohen como TE de la t de Student, y una V de Cramer sin el reporte de los valores mostrados por el Chi cuadrado, pero no se ha reportado el TE en las investigaciones con diseños pretest-postest y con grupos no equivalentes, por lo que se desconoce si las diferencias han sido grandes, medianas o pequeñas. Debido al anterior punto, coincidimos con Vargas (2011), quien considera que el psicólogo (local) valida su investigación empleando la estadística sin tomar importancia a la postura teórica, ya que, con colocar un valor numérico, para él es suficiente para hacer ciencia.

En principio, la determinación de la muestra puede parecer un proceso simple pero que su determinación y la forma de asignación puede tener consecuencias importantes sobre la conclusión de toda investigaciones (Martínez, Castellanos y Chacón, 2014). Una crítica frecuente a los estudios en psicología es la ausencia de muestras representativas, aunque la posibilidad de generalizar, dependerá del fenómeno que se estudia (Martínez et al., 2014). Esto se demuestra en que la mayoría de tesis y artículos se ha utilizado el muestreo no probabilístico, incluso otra gran porción de estudios no lo ha reportado. Este punto es interesante si se considera el uso exclusivo de la prueba de significancia estadística para concluir si se acepta o se rechaza la hipótesis planteada sin tener en cuenta la significancia práctica, ya que como se sabe, este método estadístico está fuertemente ligado a la probabilidad (Pita y Pértega, 2000). No obstante, los tesistas y los autores de artículos eligen una muestra no aleatoria para concluir unicamente en base de la significancia estadística, lo cual revela una incongruencia, un mal uso de la estadística y una interpretación errada de los valores arrojados por las pruebas aplicadas.

Es imporante destacar el frecuente uso de SPSS para analizar los datos, pues el 87,6\% de las tesis y el $47,4 \%$ de los artículos señalaron haber hecho uso de este software estadístico. En el caso de los estudios instrumentales, también refirieron haber usado SPSS para el AFE y AMOS para el AFC. Lo cual se puede deber a que son programas clásicos, comerciales, pero con limitaciones para el análisis estadístico como demanda la comunidad científica actualmente, pues, por ejemplo, no reportan tamaños de efecto para las pruebas más utilizadas como las pruebas t, la U de Mann Whitney, ANOVA, etc; además, tampoco muestran estimadores robustos. Algo rescatable del SPSS podría ser la facilidad de analizar los datos, ya que es un programa amigable para el usuario que se inicia en la estadística. No obstante, existen opciones que superan las limitaciones mencionadas y son igual de amigables como JAMOVI (Jamovi Project, 2018), que tiene módulos de estadística robusta, metaanálisis, AFE, AFC, coeficientes de confiabilidad como el omega, y son de libre distribución. Otra buena opción podría ser JASP (JASP Team, 2018), que tiene implementado el paquete Lavaan para AFC.

En la revisión de los trabajos de grado y artículos, también nos hemos podido dar 
cuenta de las limitaciones tanto métológicas como estadísticas al momento de elaborar y ejecutar un proyecto de investigación, pues se hace un mal uso de la estadística, que conlleva a resultados sesgados o errados según las recomendaciones actuales que se pueden encontrar, por ejemplo, en la APA (2010) y la AERA, APA y NCME (2014). Además, hemos encontrado plagios en las tesis de pregrado y postgrado, e incluso citas que no estaban en el formato APA solicitado por las universidades.

Por último, se hará mención a las limitaciones de la investigación. En primer lugar, el intervalo de tiempo escogido hizo que se descarte las investigaciones que se hayan podido realizar anteriormente al año 2014. Esto porque se tomó en cuenta como referencia temporal a la promulgación de la Nueva Ley Universitaria $N^{\circ} 30220$
(2014) de SUNEDU para la educación superior. En segundo lugar, no se revisaron estudios que se hayan realizado en dos universidades que en su momento tenían dentro de su oferta académica a la carrera de Psicología, debido a que éstas ya no se encuentran habilitadas, además de no encontrarse registros de estudios realizados en Cajamarca. Y finalmente, al momento de realizar la revisión, se han llevado a cabo sustentaciones públicas de tesis que podrían haber estado consideradas aquí, pero no se las incluyó porque aún no se encontraban en el repositorio digital de las universidades ni de manera física en las bibliotecas correspondientes.

\section{Conflicto de intereses}

Los autores expresan que no existe conflictos de interés al llevar a cabo la presente investigación. 


\section{Referencias}

AERA, APA \& NCME. (2014). Standards for Educational and Psychological Testing. Washington, DC: American Educational Research Association.

Alarcón, R. (2002). Estudios sobre psicología latinoamericana. Lima: Editorial Universitaria.

Alarcón, R. (2012). Psicología y testimonio personal. En H. Klappenbach, \& R. León, Historia de la psicología iberoamericana en autobiografías (pp. 21-67). Lima: Universidad Ricardo Palma.

Alarcón, R. (2013). Métodos y diseños de investigación del comportamiento. Lima: Universidad Ricardo Palma.

Alarcón, R. (2017). Historia de la psicología en el Perú. De la Colonia a la República. 2da. Edición. Lima: Universidad Ricardo Palma.

American Psychological Association (APA). (2010). Manual de Publicaciones de la American Psychological Association. zra Edición. México, D.F.: Editorial El Manual Moderno.

American Psychological Association (APA). (2010). Publication manual of the American Psychological Association. 6th Edition. Washington, DC: APA.

Arana, J. M., Mellán, J. J., \& Pérez, E. (2006). El concepto de psicología: entre la diversidad conceptual y la conveniencia de unificación. Psicología y Educación, $8(1), 111-142$.

Ato, M., López, J. J., \& Benavente, A. (2013). Un sistema de clasificación de los diseños de investigación en psicología. Anales de Psicología, 29(3), 1038-1059.

Bermudez, J. (2013). Investigacion científica en el Perú: factor crítico de éxito para el desarrollo del país. Revista Sinergia e Innovación, 1(2). Recuperado de http:// repositorioacademico.upc.edu.pe/upc/handle/10757/334666

Bunge, M. (1959). La ciencia, su método su filosofía. Buenos Aires: Editorial Siglo Veinte.

Bunge, M. (1980). Epistemología. Curso de Actualización. Barcelona: Editorial Ariel. 
Bunge, M. (1989). Ciencia y desarrollo. Buenos Aires: Siglo XX.

Bunge, M. (2009). Vigencia de la filosofía. Lima: Editorial de la Universidad Inca Garcilazo de la Vega.

Byrne, B. (2010). Structural Equations Modeling with AMOS (2nd ed.). New York: Routledge.

Cronbach, L. J., \& Shavelson, R. J. (2004). My current thoughts on coefficient alpha and successor procedures. Educational and Psychological Measurement, 64(3), 391-418. doi:10.1177/oo13164404266386

Ferrando, P., \& Lorenzo-Seva, U. (2014). El Análisis Factorial Exploratorio de los ítems: algunas consideraciones adicionales. Anales de Psicología, 30(3), 1170-1175. doi: https://dx.doi.org/10.6o18/analesps.30.3.199991

García, C. (2013). Cómo investigar en psicología. México, D.F.: Trillas.

Hidalgo, M. (2015). Investigación científica en la universidad pública peruana y su relación con el estado y empresa. Revista Quipukamayoc, 23(44), 95-101.

Jamovi Project (2018). Jamovi (Versión o.9) [Computer Software]. Recuperado de: https://www.jamovi.org

JASP Team. (2018). JASP (Version o.9)[Computer software]. Recuperado de: https:// jasp-stats.org/

Labrador, F., \& Crespo, M. (2012). Psicología clínica basada en la evidencia. Madrid: Ediciones Pirámide.

Llobell, J. P., Frías, M. D., \& Monterde i Bort, H. (2004). Tratamientos psicológicos con apoyo empírico y práctica clínica basada en la evidencia. Papeles del Psicológo, $25(87), 1-8$.

Lloret-Segura, S., Ferreres-Traver, A., Hernández-Baeza, A., \& Tomás-Marco, I. (2014). El análisis factorial exploratorio de los ítems: una guía práctica, revisada y actualizada. Anales de psicología, 30(3), 1151-1169.

Lorenzo-Seva, U., \& Ferrando, P. (2006). FACTOR: A computer program to fit the exploratory factor analysis model. Behavioral Research Methods, Instruments and Computers, 38(1), 88-91. 
Mardia, K. V. (1970). Measures of multivariate skewnees and kurtosis with applications. Biometrika, 57, 519-530.

Martínez, R., Castellanos, M., \& Chacón, J. (2014). Métodos de investigación en psicología. Madrid: EOS Universitaria.

McNeish, D. (2017). Thanks Coefficient Alpha, We'll Take It From Here. Psychological Methods. doi: http://dx.doi.org/10.1037/metoooo144

Ministerio de Educación [MINEDU]. (2016). Currículo Nacional de la Educación Básica. Ministerio de Educación. Recuperado de: http://www.minedu.gob.pe/ curriculo/pdf/curriculo-nacional-2016-2.pdf

Nueva Ley Universitaria $\mathrm{N}^{\circ}$ 30220. (9 de Julio de 2014). El peruano. Recuperadode: https:// www.sunedu.gob.pe/wp-content/uploads/2017/o4/Ley-universitaria-30220.pdf

Ochoa, L. (2011). La elaboración de una tesis de maestría: exigencias y dificultades percibidas por sus protagonistas. Revista Entornos, 24, 171-184.

Orbegoso, A. (2015). Los primeros (y olvidados) laboratorios de psicología experimental en el Perú. Revista de Psicología Universidad Católica San Pablo, 5(1), 57-68.

Piscoya, L. (1995). Investigación científica y educacional: un enfoque epistemológico. 2da. Edición. Lima: Amaru Editores.

Pita, S., \& Pértega, S. (200o). Significancia estadística y relevancia clínica. Cuadernos de Atención Primaria, 8, 191-195.

R Core Team. (2018). R: A Language and Environment for Statistical Computing. $R$ Foundation for Statistical Computing. Viena. Recuperado de: https://www.R-project.org

Rozeboom, W. (1960). The fallacy of the null-hypothesis significance test. Psychological Bulletin, 57, 416-428.

Sánchez, H. (2016). Desafíos de la investigación psicológica en el Perú ante el avance de la ciencia y la tecnología en el siglo XXI. Revista Tradición, 16, 43-54.

Sánchez, H., \& Reyes, C. (2015). Metodología y diseños en la investigación científica. 5ta. Edición. Lima: Bussiness Support Anneth SRL. 
Sánchez-Vásquez, M. J. (2014). Aspectos metodológicos en la enseñanza de la metodología de investigación en psicología. En M. J. Sánchez-Vásquez, La enseñanza de la metodología de la investigación en Psicología un acercamiento crítico a sus fundamentos y problemáticas (pp. 14-30). La Plata: Editorial de la Universidad de La Plata.

Sánchez-Villena, A. (2018). Relación entre estrés académico e ideación suicida en estudiantes universitarios. Eureka Revista Científica de Psicología, 15(1), 28-38.

Sarmiento, J. (2008). La Educación en Cajamarca: Siglo XXI. Cajamarca: Martínez Compañon Editores S.R.L.

Sarria, M., \& Silva, L. C. (2004). Las pruebas de significación estadística en tres revistas biomédicas: una revisión crítica. Revista Panamericana de Salud Pública, $15,300-306$.

Spink, P. (2009). Los psicólogos y las políticas públicas en América Latina: el big mac y los caballos de Troia. Psicoperspectivas, 8(2), 12-34.

Ventura-León, J. L. (2017). El significado de la significancia estadística: comentarios a Martínez-Ferrer y colaboradores. Salud Pública de México, 59(5), 499-500.

Recibido: 11 de abril de 2019

Aceptado: 21 de octubre de 2019 